
Revue
de l'histoire
Revue de l'histoire des religions
des religions
2| 2013
Écrire dans les pratiques rituelles de la Méditerranée antique. Identités et autorités

\title{
Fonctions de l'écriture dans les inscriptions religieuses de l'Anatolie romaine : du monumentum à l'écriture efficace
}

Functions of writing in the religious epigraphy of Roman Anatolia: from monumentum to efficacious writing

Nicole Belayche

\section{(2) OpenEdition}

Journals

Édition électronique

URL : http://journals.openedition.org/rhr/8114

DOI : $10.4000 /$ rhr.8114

ISSN : 2105-2573

Éditeur

Armand Colin

Édition imprimée

Date de publication : 1 juin 2013

Pagination : 253-272

ISBN : 978-2-200-92864-3

ISSN : 0035-1423

Référence électronique

Nicole Belayche, «Fonctions de l'écriture dans les inscriptions religieuses de l'Anatolie romaine: du monumentum à l'écriture efficace », Revue de l'histoire des religions [En ligne], 2 | 2013, mis en ligne le 01 juin 2016, consulté le 19 avril 2019. URL : http://journals.openedition.org/rhr/8114; DOI : 10.4000/ rhr.8114 


\section{Fonctions de l'écriture dans les inscriptions religieuses de l'Anatolie romaine: du monumentum à l'écriture efficace}

Les raisons sociopolitiques avancées pour expliquer l'explosion de l'écrit sur pierre à l'époque impériale n'épuisent pas les ressorts de certaines pratiques régionales de l'épigraphie religieuse. En Anatolie, l'ensemble des stèles "de confession" relève de l'epigraphic habit pour ses deux fonctions mémorielle et publicitaire ou honorifique; mais ce sont les dieux et leurs pouvoirs que les textes immortalisent, pas les dédicants. Les textes gravés insistent tout spécialement sur l'écriture, autorisée par les prêtres, comme vecteur de l'exaltation des dieux: réclamés par les dieux eux-mêmes, ils confinent à des proclamations catéchétiques. Enfin, dans le cours des rituels qui en appellent à la justice des dieux, l'écriture joue un rôle performatif.

\section{Functions of writing in the religious epigraphy of Roman Anatolia:} from monumentum to efficacious writing.

Studies on increasing of writing during the imperial period concluded to socio-political reasons in the majority. These reasons do not enlighten properly some local practices in religious epigraphy. In Anatolia, the group of "confession"-steles follows the line of the "epigraphic habit" for two of its functions: the memorial one and the media or honorific one. And yet they immortalize the gods and their powers, and not the devotees. Engraved texts particularly emphasize writing, authorized by the priests, as a vector for the exaltation of the divine: required by the gods themselves, they reach a form of catechetical discourse. Finally, when rituals call for divine justice, writing has a performative function. 


\section{«Primus litteras Mercurius enarrauerit: necessarias confitebor et commerciis rerum et nostris erga Deum studiis». Tertullien, De corona $8,2^{1}$.}

Depuis une génération au moins, historiens et épigraphistes de l'époque romaine n'ont cessé de s'interroger sur ce que Ramsay MacMullen a appelé, dans un article éponyme, the epigraphic habit, la pratique épigraphique ${ }^{2}$. Emporté par l'intérêt que les AngloSaxons portaient à la literacy dans son sens premier de lettrisme ${ }^{3}$, et en se fondant sur des traitements statistiques, entre autres chronologiques, réalisés sur les centaines de milliers d'inscriptions latines qui nous sont parvenues ${ }^{4}$, l'historien américain cherchait à comprendre l'explosion de textes gravés, de natures très diverses et dans des milieux qui ne le sont pas moins, à partir des Antonins et plus encore sous les Sévères, entre 160 et 230 environ. Remarquant, après et avant d'autres, que le nombre de documents écrits sur pierre est moins un indicateur du niveau de l'alphabétisation ${ }^{5}$ que de l'image de l'écrit dans une société, R. MacMullen a cherché à

1. «Le premier Mercure a enseigné les lettres: je les considère comme nécessaires tant pour le commerce des choses que pour nos devoirs envers Dieu ».

2. Ramsay MacMullen, "The Epigraphic Habit in the Roman Empire», The American Journal of Philology 103, 1982, p. 233-246.

3. Cf. William V. Harris, Ancient Literacy, Harvard UP, 1989, et la discussion sur l'ampleur du phénomène et sa définition dans John H. Humphrey éd., Literacy in the Roman World, London (Journal of Roman Archaeology Suppl. 3), 1991, en part. Mireille Corbier, «L'écriture en quête de lecteurs» et Alan K. Bowman, «Literacy in the Roman Empire: mass and mode», p. 99-132; Shirley Werner, «Literacy Studies in Classics. The Last Twenty Years», Ancient Literacies. The Culture of Reading in Greece and Rome, William A. Johnson et Holt N. Parker éds., Oxford, 2009, p. 333-382. La référence anthropologique de ces travaux est Jack Goody, The Domestication of the Savage Mind, Cambridge UP, 1977.

4. Stanislaw Mrozek, «À propos de la répartition chronologique des inscriptions latines dans le Haut-Empire», Epigraphica 35, 1973, p. 113-118 et 50, 1988, p. 61-64.

5. Ramsay MacMullen, «The Epigraphic Habit», p. 237. Pour l'apport de l'épigraphie à l'évaluation de l'alphabétisme, cf. William V. Harris, «Literacy and epigraphy. I», Zeitschrift für Papyrologie und Epigraphik 52, 1983, p. 87-111 (repris largement dans son livre cité supra n. 3). Pour la ville de Rome, Mireille Corbier («L'écriture dans l'espace public romain», L'Urbs. Espace urbain et histoire (Ir siècle av. J.-C.-III siècle ap. J.-C.), Rome [CEFR 98], 1987, p. 27-60) concluait à «une alphabétisation pauvre, largement répandue» (p. 59). 
dépasser le rôle mémoriel élémentaire que joue toute écriture sur support dur à toute époque. Il a relié l'epigraphic habit à un effet de la romanisation et à ce qu'il définissait pour conclure comme the sense of audience ${ }^{6}$.

Sa réflexion portait principalement sur le monde latinophone ${ }^{7}$. Des recherches similaires ont fleuri pour le monde impérial hellénophone; mais leurs prémisses devaient être différentes du fait du contexte historico-culturel de la Méditerranée orientale. Dans la vie civique, les Grecs étaient habitués à la pratique régulière de l'épigraphie depuis l'époque classique, Aristote considérant que les grammata étaient nécessaires aux politikai praxeis ${ }^{8}$. À l'époque hellénistique, à partir de la fin du III ${ }^{\mathrm{e}}$ siècle - début du $\mathrm{II}^{\mathrm{e}}$ siècle avant notre ère, malgré la perte progressive de leurs souverainetés, les cités grecques retrouvent un dynamisme qui génère le développement d'élites locales qui s'illustrent ou sont illustrées sur la pierre. Le phénomène s'intensifie lorsque Rome installe son imperium et que de véritables stratégies de communication et d'image sont mises en place par les cités et leurs élites dans les rapports de force entre poleis et avec le pouvoir romain ${ }^{9}$. Toutefois, les explications sociopolitiques, voire juridiques ${ }^{10}$, de l'explosion de la pratique

6. Ramsay MacMullen, «The Epigraphic Habit», p. 246.

7. Voir aussi Mary Beard, «Writing and religion: Ancient Literacy and the function of the written word in Roman religion», dans John H. Humphrey éd., Literacy in the Roman World London (Journal of Roman Archaeology Suppl. 3), 1991, p. 35-58.

8. Aristote, Politique VIII, 3 [1338 a]. Cf. F.D. Harvey, «Literacy in Athenian Democracy », Revue des Études Grecques 79, 1966, p. 585-635 (sans attention à l'épigraphie religieuse) et Marcel Detienne éd., Les savoirs de l'écriture. En Grèce ancienne, Lille, 1992.

9. Cf. l'article célèbre de Louis Robert, «La titulature de Nicée et de Nicomédie. La gloire et la haine», Harvard Studies in Classical Philology 81, 1977, p. 1-39, republié dans Id., Choix d'écrits, Denis Rousset éd., Paris, 2007, p. 673-703. Cf. Nicole Belayche, «Un dieu est né... à Stratonicée de Carie (IStratonikeia 10)», dans Christophe Batsch \& Madalina Vartejanu-Joubert éds., Manières de penser dans l'Antiquité méditerranéenne et orientale. Mélanges offerts à Francis Schmidt, Leiden (Suppl. Journal of Study of Judaism 134), 2009, p. 193-212. Plus généralement, Anna Heller, "Les bêtises des Grecs». Conflits et rivalités entre cités d'Asie et de Bithynie à l'époque romaine (129 a.C.-235 p.C.), Bordeaux (Ausonius, Scripta Antiqua 17), 2006 et Yves Lafond, La mémoire des cités dans le Péloponnèse d'époque romaine, II siècle avant J.-C - III siècle après J.-C., Rennes, 2006.

10. Par ex., Elisabeth A. Meyer, «Explaining the Epigraphic Habit in the Roman Empire: the Evidence of Epitaphs », Journal of Roman Studies 80, 1990, p. 74-96, a relié de façon convaincante la pratique des gravures d'épitaphes avec 
épigraphique rendent insuffisamment compte de certaines pratiques régionales ou spécifiques de l'épigraphie religieuse ${ }^{11}$, pourtant contemporaines. Commanditées par des personnes privées tout en visant un public, comme tout document gravé, les ressorts de leur gravure relèvent pour partie seulement de ceux de l'epigraphic habit civique; ils résident, à mon sens, dans le rôle monarchique reconnu aux puissances divines ${ }^{12}$.

L'ensemble documentaire ici privilégié est cohérent à la fois géographiquement et chronologiquement (pour l'essentiel du $\mathrm{II}^{\mathrm{e}}$ siècle au milieu du $\mathrm{III}^{\mathrm{e}}$ siècle environ); il est également cohérent épigraphiquement, sociologiquement et religieusement, ce qui permet de comparer des séries. Les textes concernés sont connus sous l'appellation de «stèles de confession»/ Beichtinschriften ${ }^{13}$. Ils proviennent d'une zone géographique bien circonscrite: les plateaux enclavés et ingrats de la Lydie et la Phrygie occidentale, entre l'Hermos et le Méandre, pays brûlés pour la Lydie Katakekauménè ${ }^{14}$, qui comptent parmi les régions les moins urbanisées de l'Anatolie centre-occidentale ${ }^{15}$. À l'écart des grands courants d'échange (qui passaient plus à l'ouest, ou bien au nord et au sud) ${ }^{16}$, la présence romaine y était lâche. Cependant le tissu civique s'y est densifié depuis Auguste et des établissements impériaux ont favorisé l'intégration des populations locales ${ }^{17}$, à en

le statut de citoyen romain (cf. la comparaison entre Thessalonique et Athènes, p. 92).

11. Souvent des dédicaces votives et des imprécations, par ex. William V. Harris, Ancient Literacy, p. 218-221.

12. Cette étude est partie de la conclusion («La narration écrite, une eulogia arétalogique») de mon article «Les stèles dites de confession: une religiosité originale dans l'Anatolie impériale? », dans Lukas de Blois, Peter Funke, Johannes Hahn éds., The Impact of Imperial Rome on Religions, Ritual and Religious Life in the Roman Empire, Leiden-Boston, 2006, p. 66-81.

13. Georg Petzl, Die Beichtinschriften Westkleinasiens, Bonn, 1994 (Epigraphica Anatolica 22), dorénavant: Petzl 1994. Une nouvelle édition est en préparation du fait des nombreuses publications d'inédits depuis 1994.

14. Cf. Peter Thonemann, The Maeander Valley: A Historical Geography from Antiquity to Byzantium, Cambridge-New York, 2011.

15. Cf. Stephen Mitchell, Anatolia. Land, Men and Gods in Asia Minor. I. The Celts and the Impact of Roman Rule, Oxford, 1993, p. 190, carte 11.

16. Une seule grande voie de Tabala à Dorylaeum, cf. Stephen Mitchell, Anatolia, I, p. 124-132.

17. Cf. les dédicaces aux Theoi Patrioi et à l'empereur, Hasan Malay, Greek and Latin Inscriptions in the Manisa Museum, Vienne (Denkschrift d. Österr. Akad. d. Wissensch., Phil.-Hist. Kl. 237, ETAM 19), 1994, n 192, 193 \& 195 
juger par l'anthroponymie. Les dévots commanditaires des textes sont des villageois petits possédants, agriculteurs et éleveurs ${ }^{18}$, dont l'accès aux pierres était facilité par la présence de nombreuses carrières, en particulier celles de pavonazzetto à Dokimeion ${ }^{19}$. Cette donnée peut fournir un premier élément d'explication à la masse d'inscriptions dans des milieux ruraux et villageois qui ne sont pas ceux de l'epigraphic habit ${ }^{20}$. Le relatif enclavement de ces régions aura contribué à maintenir les identités culturelles locales, accrochées à une kyrielle de petits sanctuaires, consacrés à autant de divinités à épiclèses topiques derrière les appellations génériques de Mên, Meter et Zeus ${ }^{21}$. D'après les inscriptions, ces divinités sont des seigneurs des lieux qui ne cessent de manifester leur puissance souveraine ${ }^{22}$ sur les communautés villageoises ${ }^{23}$.

Les textes dits de confession sont longs et narratifs, une prolixité

(Hadrien et Antonin le Pieux); une imprécation par tous les dieux du peuple romain, Johann Strubbe, APAI EПITYMBIOI. Imprecations against Desecrators of the Grave in the Greek Epitaphs of Asia Minor, Bonn (IK 52), 1997, $\mathrm{n}^{\circ}$ 5, 11. 4-5; et les combats de gladiateurs à Sattai, Louis Robert, Les Gladiateurs dans l'Orient grec, Paris, 1940, $\mathrm{n}^{\circ}$ 134-136 et Hellenica VIII, Paris, 1950, p. 65-66.

18. Cf. une historiette dans Petzl 1994, $\mathrm{n}^{\circ}$ 68. Cf. Tommaso Gnoli, John

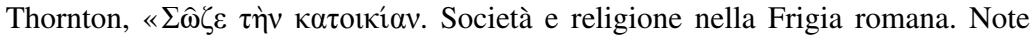
introduttive », dans Roberto Gusmani, Mirjo Salvini, Pietro Vannicelli éds., Frigi e Frigio. Atti del I Simposio Internazionale, Roma, 16-17 ottobre 1995, Rome, 1997, p. 159-162, 182 pour les bœufs.

19. Michel Christol, «Les carrières de Dokimeion à l'époque sévérienne», Epigraphica 53, 1991, p. 113-174 et Thomas Drew-Bear, «Nouvelles inscriptions de Dokimeion», Mélanges de l'École française de Rome. Antiquité 106, 2, 1994, p. 747-844.

20. Sur la relation entre illettrisme et milieux ruraux et féminins dans l'Athènes des $\mathrm{V}^{\mathrm{e}}$ et $\mathrm{IV}^{\mathrm{e}}$ siècles, F.D. Harvey, «Literacy in Athenian Democracy», et les observations méthodologiques de William V. Harris, Ancient Literacy, p. 22-24 et 190-193.

21. Cf. Maria Paz de Hoz, Die lydischen Kulte im Lichte der griechischen Inschriften, Bonn (Asia Minor Studien 36), 1999. Certains de ces sanctuaires ont été localisés, voire fouillés, mais pas toujours publiés (cf. pour celui de Mên d'Artemidoros et de sa Mètèr à Axiotta / Magazadamlari, l'annonce de la publication dans Peter Herrmann \& Hasan Malay, New Inscriptions from Lydia, Wien (ETAM 24), 2007, p. 76 n. 110.

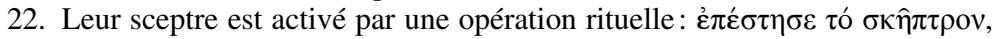
Petzl 1994, n 35, 11. 12-13.

23. Cf. Peter Herrmann, «Men, Herr von Axiotta», dans Studien zur Religion und Kultur Kleinasiens, Leiden (EPRO 66/2), 1978, p.415-423 et Nicole Belayche, «"Au(x) dieu(x) qui règne(nt) sur...". Basileia divine et fonctionnement du polythéisme dans l'Anatolie impériale», dans Annie Vigourt, Xavier Loriot et al. éds., Pouvoir et religion dans le monde romain. En hommage à Jean-Pierre Martin, Paris, 2005, p. 257-269. 
qui les distingue des dédicaces religieuses habituelles. Ils sont construits selon une structure stéréotypée qui se décompose, à mon sens $^{24}$, en quatre étapes qui suivent la narration orale que le dévot a faite de cette tranche de sa vie, sans doute dans le sanctuaire. Après l'acclamation de la (ou des) divinité(s), le dévot rapporte un malheur qui l'a frappé, lui ou sa famille, voire son bétail ${ }^{25}$, et qu'il présente comme un châtiment des dieux, la rançon d'une faute $(1: \kappa o \lambda \alpha \dot{\zeta} \zeta)$. Il en fait alors l'aveu $(2: \delta \mu \nu \lambda o \gamma \hat{\omega}$, une fois $\mu \alpha \rho \tau v \rho \hat{\omega})^{26}$. Après une expiation rituelle qui le réconcilie avec la

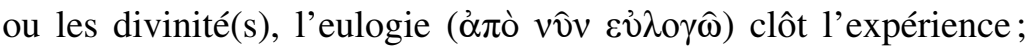
cette quatrième et dernière étape insiste sur l'écriture de la stèle:

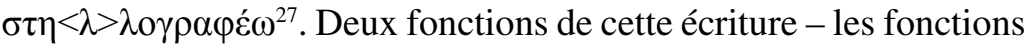
mémorielle et publicitaire ou honorifique - recoupent celle de l'epigraphic habit, mais l'écriture a aussi dans ces textes des fonctions propres. Renversant ouvertement la fonction honorifique, les textes sont présentés comme une exigence des dieux eux-mêmes de façon que leur puissance soit exaltée; d'où des proclamations de forme catéchétique, qui sont originales en monde polythéiste ${ }^{28}$. En outre, l'écriture intervient dans le cours même de ces expériences religieuses singulières, comme outil performatif lorsqu'un rituel fait appel à la justice des dieux.

\section{FonCTION MÉMORIELLE ET PUBLICITAIRE}

Avant toute fonction singulière, les textes dits de confession répondent banalement à la fonction mémorielle ${ }^{29}$ et publicitaire de

24. Cf. aussi Richard Gordon, «Raising a sceptre: confession-narratives from Lydia and Phrygia », Journal of Roman Archaeology 17, 2004, p. 189 (provocation, punishment, anagnorisis, resolution). Pour une présentation générale, Nicole Belayche, "Résumé des conférences et travaux», Annuaire de l'EPHE, section des Sciences religieuses 111, 2002-2003, p. 238-242.

25. MAMA IV, 286.

26. Des parallèles dans les sanctuaires thérapeutiques, par ex. IG XIV 966

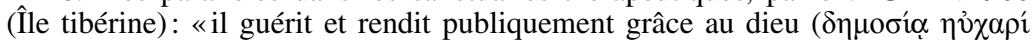
$\sigma \tau \eta \sigma \varepsilon v) »$. Cf. M.P.J. Dillon, «The Didactic Nature of the Epidaurian Iamata», Zeitschrift für Papyrologie und Epigraphik 101, 1994, p. 239-260.

27. Petzl 1994, $\mathrm{n}^{\circ} 37$. L'ordre indiqué n'est pas toujours respecté, ni toutes les étapes mentionnées (par ex. Petzl 1994, $\mathrm{n}^{\circ} 112$ ).

28. Dans sa liste, certes générale, des fonctions religieuses de l'écriture, William V. Harris, Ancient Literacy, p. 27, ne la signale pas.

29. Diodore de Sicile XII, 13, 2: «Par elle [l'écriture] seule, en effet, les morts subsistent dans la mémoire des vivants ». 
la gravure sur pierre, donc une fonction de communication avec tous les partenaires de la collectivité, hommes comme dieux ${ }^{30}$. Engraphein ou anagraphein en stèllè est un acte quotidien dans la vie de la cité pour publier durablement un fait ou une décision ${ }^{31}$. Dans la vie religieuse publique comme privée, les inscriptions

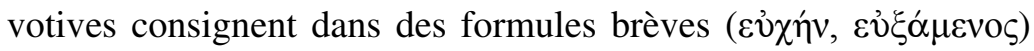
l'acquittement des vœux. Une épigramme de Callimaque exprime de façon très explicite le recours qu' on pouvait y faire face au dieu:

Sache, Asclépios, que tu as reçu la dette qu'Akeson avait contractée à la suite de son vœu pour sa femme Demodikè. Mais si tu oublies et réclames à nouveau ton dû, la tablette dit ( $\varphi \eta \sigma i ̀$ [...] ó $\pi i ́ v \alpha \xi)$ qu'elle

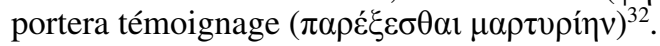

La pratique de l'accrochage de pinakes - des tableaux de bois qui pour leur part ne se sont pas conservés - sur les murs ou dans les arbres, portant des ex-voto sous forme de textes ou d'images, a déjà été bien étudiée ${ }^{33}$.

La stèle comme objet affiche sa destination mémorielle: elle est un marturion comme vient de le dire Akeson ${ }^{34}$, un monumentum en latin, qui désigne tout à la fois l'objet et la consignation qu'il porte de l'expérience faite du pouvoir des dieux ${ }^{35}$. Aussi fait-elle mémoire du dieu autant que de son dévot ${ }^{36}$. Les stèles étaient probablement

30. Cf. Diodore de Sicile XII, 13, 2: «Ceux qu'une grande distance sépare communiquent, en écrivant, comme s'ils étaient proches».

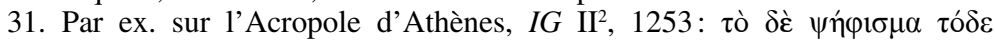

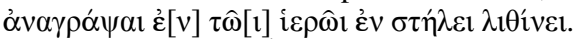

32. Callimaque, Épigramme 55 (= Anthologie Palatine VI, 147).

33. Paul Veyne, «'Titulus praelatus' : offrande, solennisation et publicité dans les ex-voto gréco-romains», Revue archéologique 2, 1983, p. 281-300 et Folker T. Van Straten, Hiera Kala. Images of Animal Sacrifice in Archaic and Classical Greece (Religions of the Graeco-Roman World 127), Leiden, 1995, p. 57-58. Pour les sanctuaries thérapeutiques, Emma J. Edelstein, Ludwig Edelstein, Gary B. Ferngren, Asclepius. Collection and Interpretation of the Testimonies, Baltimore, 1945 [1998], $\mathrm{n}^{\circ} 423$ A III, p. 222: «Regardant les tablettes exposées dans le

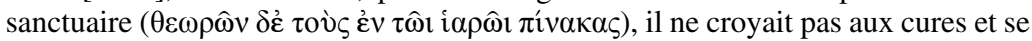
moquait des inscriptions (

34. Voir aussi Petzl 1994, $n^{\circ} 9$, 1l. 13-14 \& n ${ }^{\circ}$ 1, ll. 7-8 et Irene Diakonoff, «Artemidi Anaeiti anestesen. The Anaeitis-dedications in the Rijksmuseum van Oudheden at Leyden and related material from Eastern Lydia. A reconsideration », BABESCH 54, 1979, p. 151 n 32 (des dévots d'Artémis Anaitis et Mèn Tiamos

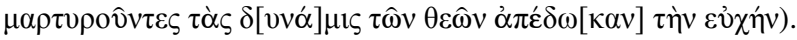

35. Cf. Gabriel Sanders, «Texte et monument: l'arbitrage du Musée épigraphique», Il Museo epigrafico, Faenza, 1984, p. 104.

36. Cf. Paul Veyne, «'Titulus praelatus'», p. 294: «La piété devient une occasion pour le fidèle de se célébrer lui-même». 
fichées en terre ou sur un socle dans les cours des sanctuaires, à en juger par les épais tenons qui étaient ménagés ${ }^{37}$. Conformément aux procédures votives traditionnelles, et même s'il est rare que le contrat votif soit explicité, au vu des nombreux parallèles où la divinité réclame l'érection de la stèle, il est probable que le fidèle promettait la stèle inscrite en action de grâce:

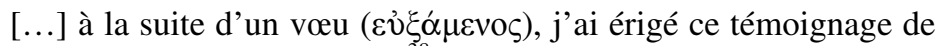

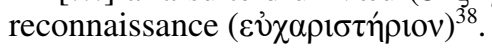

D'ailleurs, les dieux ne manquent pas de réclamer leur dû en punissant les ingrats. Prepousa, par exemple, avait promis ( $\varepsilon \hat{\jmath} \xi \varepsilon \tau o$ ) d'inscrire une stèle $(\sigma \tau \eta \lambda \lambda o \gamma \rho \alpha \varphi \hat{\eta} \sigma \alpha l)$ si les dieux protégeaient la santé de son fils;

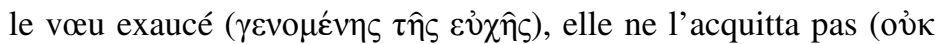
$\dot{\alpha} \pi \varepsilon ́ \delta \omega \kappa \varepsilon v)$; maintenant, le dieu a réclamé le vœu (⿳亠丷厂

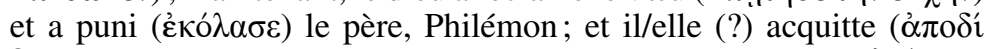

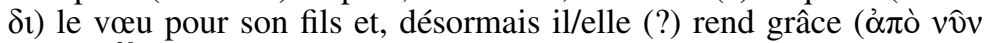
$\varepsilon \hat{\jmath} \lambda \circ \gamma \hat{\imath})^{39}$.

Même lorsque l'acquittement du vœu n'est que retardé, la divinité impatiente «a ordonné d'écrire les puissances du dieu ${ }^{40}$.

Pourtant, même lorsqu'ils comportent un épisode votif comme

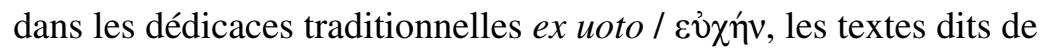
confession ne se limitent pas à témoigner durablement d'un rituel acquitté. Par-delà l'archivage, la stèle inscrite renforce la ritualité du discours ${ }^{41}$; elle sert d'eulogia pérenne, d'hommage perpétuel à la divinité après l'expérience de leurs puissances:

[...] ayant été punie, j'ai avoué la faute et j'ai consacré l'action de

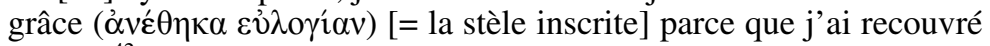
la sante ${ }^{42}$.

37. Par ex. Petzl 1994, p. 20.

38. Petzl 1994, $\mathrm{n}^{\circ} 76$ (en 235-236).

39. Petzl 1994, $\mathrm{n}^{\circ} 62$; cf. aussi ${ }^{\circ} 8$, où, en dépit des lacunes, on comprend

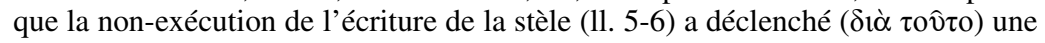
réaction des dieux.

40. Petzl 1994, ${ }^{\circ} 65$ 11. 6-7; pour un cas similaire, avec expression explicite du rapport de causalité, Peter Herrmann, Hasan Malay, New inscriptions, ${ }^{\circ}$ 55, 11. 8-10 (trad. p. 82): «Because of this I erected the stele and wrote on it the manifestations of the god's powers».

41. Cf. Thomas N. Habinek, "Situating Literacy at Rome», dans Ancient Literacies, p. 114-140, 123 pour «the ritualization of speech».

42. Petzl 1994, 43, 11. 5-8. Sur l'origine du terme eulogia, Hasan Malay, «A praise of Men Artemidorou», Epigraphica Anatolica 36, 2003, p. 15-16. 
Comme l'écrivait $\mathrm{P}$. Veyne il y a déjà trente ans, «la publicité

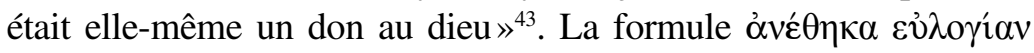

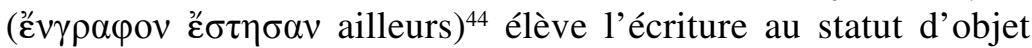
offert. Les signes inscrits ont un prestige en soi, indépendant du message délivré. Les très rares parallèles qu'on peut invoquer appartiennent à des contextes voisins des «stèles de confession»: des inscriptions funéraires ${ }^{45}$ (qui utilisent aussi l'écriture à des fins performatives, nous le verrons) et une inscription honorifique

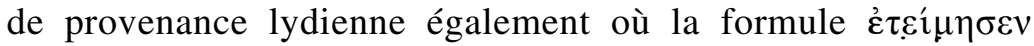

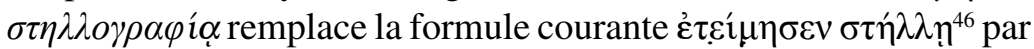
laquelle on honorait la timè des dieux ou des hommes. À Amorgos (IG XII 7, 259), un texte déclare «consacre[r] l'inscription (

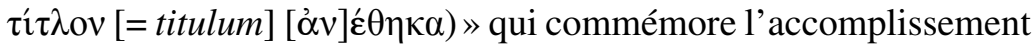
de mystères ancestraux aux dieux.

À la manière des textes arétalogiques, la fonction d'eulogia perpétuelle explique pourquoi les stèles déroulent des récits circonstanciés et attentifs à la temporalité de l'expérience complexe des dieux qui a été vécue ${ }^{47}$. Une telle précision pourrait ne faire que refléter la transcription du récit proféré oralement. Mais la rationalité de la construction des textes ${ }^{48}$, leur caractère stéréotypé, l'accent mis sur les mêmes étapes, leur tarification même (nous le verrons), portent la trace d'un encadrement étroit par les autorités sacerdotales, à la façon des iamata d'Épidaure qui jouaient en même temps le rôle d'archives médicales ${ }^{49}$.

43. Paul Veyne, «'Titulus praelatus'», p. 298.

44. Petzl 1994, 70, 1. 8.

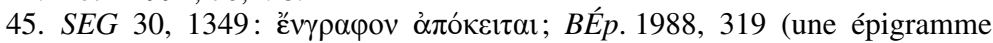

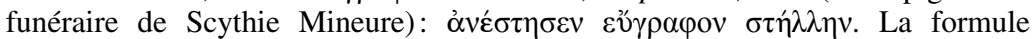

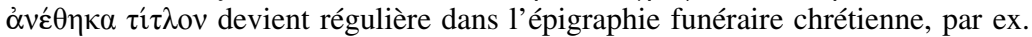
IGVR II, 1024 (cf. aussi 517) ou MAMA VII, 80.

46. Respectivement: Hasan Malay, Greek and Latin Inscriptions in the Manisa Museum, $\mathrm{n}^{\circ}$ 36, 11. 3-4 (c'est moi qui souligne); et SEG 27, 785 (Lydie).

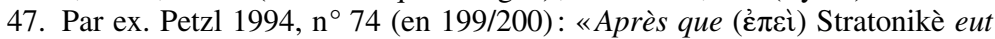

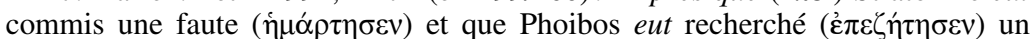

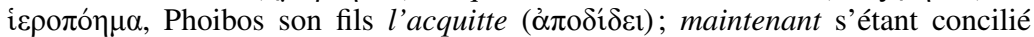

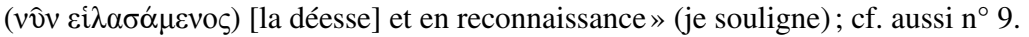

48. Richard Gordon, «Raising a scepter», p. 185 n. 38, note très justement: «The excess of causal and sequential words implies a special effort to emphasize the coherence of the elements of the narrative presented».

49. Cf. Strabon VIII, 6, 15: «Son sanctuaire est rempli en permanence de

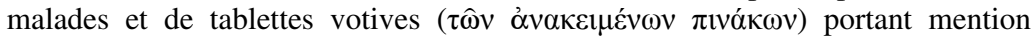

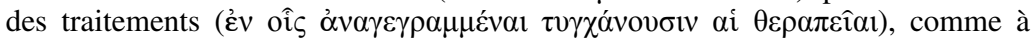
Cos et Tricca»; Pausanias II, 27, 3 (trad. Loeb Classical Library): «Within the 
Les textes dits de confession répondent donc à une fonction mémorielle classique, par-delà des nuances propres. S'y ajoute une fonction moins habituelle, celle d' afficher les règles communautaires. Rappelons d'abord qu'il serait erroné de faire équivaloir ruralité et illettrisme, comme les bergers d'Arcadie imaginés par le peintre Poussin en montrent déjà une magnifique intuition. Pour preuve, une des historiettes rapporte que des villageois cherchaient à abuser de deux orphelins «en leur arrachant des engagements écrits ( $\alpha \rho o ́ v \tau \omega v$ ह̌ $\gamma \gamma \rho \alpha \varphi \alpha) \gg^{50}$. Ces milieux lydiens et phrygiens revendiquaient aussi, pour les hommes du moins, la culture de la paideia. Sur les reliefs des stèles funéraires phrygiennes, les défunts sont régulièrement représentés avec le calamos, la tablette et le volumen, tandis que les défuntes sont accompagnées d'objets de toilette et de filage ${ }^{51}$. Ces reliefs sont évidemment bien trop stéréotypés pour dire quoi que ce soit du lettrisme réel de ces hommes, mais ils expriment un horizon culturel de référence. Dans ces bourgs (kômai) et villages qui appartiennent aux territoires des cités, les inscriptions enseignent que les petits sanctuaires ruraux qui abritaient les procédures rituelles examinées jouissaient d'une autonomie religieuse et surtout juridique pour des affaires intra-communautaires non criminelles. Les dieux - c'est-à-dire les sanctuaires - sont jaloux de prérogatives remontant à l'époque pré-civique probablement, au point de punir une femme parce qu'elle avait fait appel aux autorités civiles plutôt qu'à leur justice pour régler un différend ${ }^{52}$. Ce sont les dieux qui édictent, ou au moins garantissent, les règles collectives. En inscrivant leurs mésaventures tragiques - tant la justice des dieux est implacable -, les dévots contribuent donc à rappeler aux villageois les règlements: l'inviolabilité et les limites d'un bois sacré $^{53}$ ou bien la sûreté des biens personnels dans un bain public.

enclosure stood slabs: in my time, six remained, but of old they were more. On them are inscribed the names of both the men and the women who have been healed by Asclepios, the disease also from which each suffered and the means of

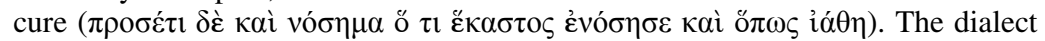
is Doric ».

50. Petzl 1994, $\mathrm{n}^{\circ} 35$ (en 210-211).

51. Par ex. MAMA X 5, 34, 430.

52. Pour des conflits d'autorité entre un sanctuaire et la justice civile, Petzl $1994, n^{\circ} 49$ et 114 .

53. Petzl 1994, $\mathrm{n}^{\circ} 76$ et Louis Robert, «Documents d'Asie Mineure. 2. Zeus des Chênes Jumeaux », Bulletin de Correspondance Hellénique 107, 1983, p. 516: «un avertissement». Cf. Hasan Malay, Manisa $\mathrm{n}^{\circ} 24$ («Lex sacra mentioning a 
Le texte d'une stèle offerte par un voleur s'ouvre par l'acclamation de Mên Axiottènos Seigneur ( $\beta \alpha \sigma \imath \lambda \varepsilon v ́ \omega v)$ de Tarsi, dont la figure gravée écrase la composition sur un tiers de l'espace:

Alors que le sceptre avait été dressé, pour le cas où quelqu'un volerait quelque chose dans le bain (public), un manteau ayant été dérobé, le dieu fut irrité contre le voleur et fit qu'au bout de quelque temps celui-ci lui remit le manteau et avoua. Cela étant, le dieu ordonna par les soins d'un messager que le manteau fût vendu et que les puissances fussent inscrites sur la stèle $(\sigma \tau \eta \lambda \lambda o \gamma \rho \alpha \varphi \hat{\eta} \sigma \alpha l \tau \grave{\alpha} \varsigma$ $\delta v v \alpha ́ \alpha \varepsilon 1 \zeta)^{54}$.

Les stèles font office d'affichage public; il n'est donc pas fortuit de retrouver un même accent mis sur l'écriture (avec les mêmes formules de «stèle inscrite» ou «inscrire la stèle») dans des textes à portée juridique comme les affranchissements d'esclaves auprès d'un temple ${ }^{55}$. Les faits divers individuels sont autant d'occasion de graver dans la pierre des règlements religieux, par exemple en 166-167 celui qui établit comment se dégager d'un serment et qui subordonne le droit d'écrire la stèle au paiement d'une amende.

Pour que les serments par le nom d'Axiottènos soient levés, [on fera] en sorte que le délieur des serments acquitte 175 deniers; en outre, sur cette somme, il retiendra le prix qu'Axiottènos a demandé

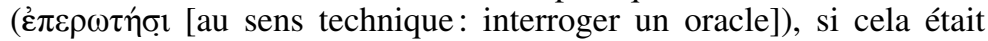

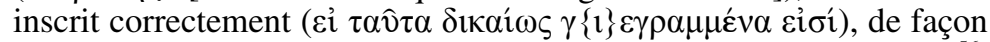

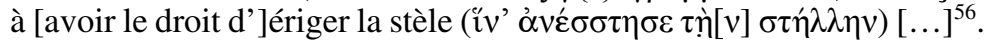

Une part de l'amende - 50 deniers d'après un autre texte (Petzl 1994, $\mathrm{n}^{\circ}$ 46), soit $30 \%$-, est déterminée par le dieu dans une communication de type divinatoire dont les modalités nous

spring and a fire»). Un parallèle à Chios ( $\left.L S C G \mathrm{n}^{\circ} 116\right)$ où la règlementation

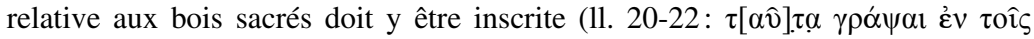
óं $\lambda \sigma \varepsilon \sigma \iota v)$.

54. Petzl 1994, 3 (traduction partielle de Louis Robert, «Documents d'Asie Mineure. 2», p. 519). Cf. Angelos Chaniotis, «'Tempeljustiz' im kaiserzeitlichen Kleinasien. Rechtliche Aspekte der Sühneinschriften Lydiens und Phrygiens», dans Gerhard Thür, Julie Vélissaropoulos-Karakostas éds., Symposion 1995. Vorträge zur griechischen und hellenistischen Rechtsgeschichte (Korfu, 1995), Köln, 1997, p. 366-367 et Georg Petzl, Die Beichtinschriften im römischen Kleinasien und der Fromme und der Gerechte Gott, Nordrhein-Westfälische Akademie der Wissenschaften, Vorträge G 355, Opladen, 1998, p. 9-11.

55. Par ex. SEG 36, 590, 1. 37. Cf. aussi dans une négociation territoriale entre

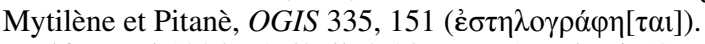

56. Petzl 1994, $n^{\circ} 58,11.9-20$ et p. 71 : «damit (?) er eine Stele aufstellt»; cf. aussi, $n^{\circ} 8,1.7$. 
échappent, hélas. Elle sert à payer la fabrication de la stèle, confectionnée, ou seulement supervisée, par les prêtres puisqu'elle doit être inscrite $\delta 1 \kappa \alpha i \omega \varsigma$, c'est-à-dire conformément aux règles et, donc, en relatant les étapes de la procédure. Dans un autre cas (Petzl 1994, n 46), la somme rendue au double par les fils d'un délinquant

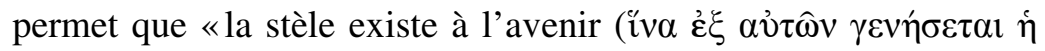
$\sigma \tau \dot{\eta} \lambda \lambda \eta) »$.

La stèle inscrite objective donc la clôture de l'affaire prononcée par le dieu lui-même. Puisqu'elle entre dans le processus de réconciliation avec le dieu, son écriture ne peut pas être laissée à la liberté du dévot. Destinée à être dressée dans le sanctuaire, et à plus forte raison lorsque les dieux l'ont réclamée, les prêtres doivent en encadrer la réalisation, d'où leur construction stéréotypée. Les textes inscrits relatent, certes, des expériences singulières, mais ils ne sont pas le produit d'un élan religieux spontané et gracieux: ils fixent des récits autorisés et nécessaires.

\section{LA «PRAGMATIQUE » GLORIFICATRICE DE L’ÉCRITURE}

Par-delà la réification de l'écriture qu'on vient de souligner, le dépouillement de la base électronique des inscriptions grecques du Packard Institute ${ }^{57}$ montre que le corpus des stèles dites de confession se distingue par l'emploi d'expressions redondantes relatives à l'écriture de la stèle. La succession des deux locutions stè(l)lographein et engraphein manifeste le souci d'exalter la puissance des dieux ${ }^{58}$. À Philadelphie de Lydie, la dédicante, considérée comme perdue par les hommes ${ }^{59}$, écrit simplement:

elle a fait inscrire la stèle et elle a dressé cet écrit et ils rendent

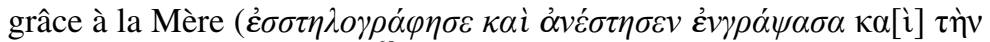
$\tau \varepsilon \kappa о \hat{\sigma} \sigma \alpha<\nu>\varepsilon \hat{\jmath} \lambda \circ \gamma \circ \hat{0} \sigma \alpha v)^{60}$.

L'écriture des souffrances du dévot, tant qu'il n'a pas avoué la faute et ne s'est pas réconcilié avec les dieux, objective la

57. http://epigraphy.packhum.org.

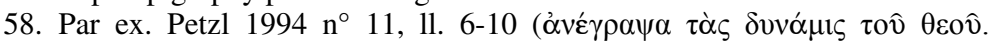

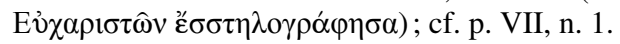

59. Comme sur beaucoup d'inscriptions de sanctuaires thérapeutiques, par ex. $I G V R$ I, $148=I G \mathrm{XIV}, 966$ ( $\mathrm{II}^{\mathrm{e}}$ siècle).

60. Petzl 1994, n 96, 11. 4-6 (je souligne). 
grandeur des dieux qui s'exprime dans leur puissance justicière - leur nemesis -. Un impie qui avait manqué aux règles rituelles en consommant des viandes non sacrifiées

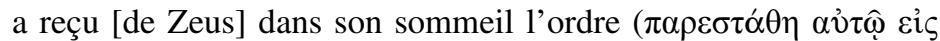

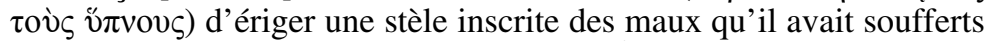

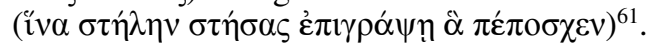

Aussi l'écriture de la stèle est-elle dans plusieurs cas la seule exigence du dieu ${ }^{62}$. Ovide déjà dans les Pontiques (I, 1, 55-58) écrivait que les dieux se réjouissaient à de telles déclarations :

Talia caelestes fieri praeconia gaudent, (Les dieux aiment que l'on fasse de telles proclamations,)

ut sua quid ualeant numina teste probent. (car elles prouvent la vigueur de leur divinité.)

Un dévot à qui les dieux réclamaient d'ériger deux statues et qui tardait à s'exécuter reçut un châtiment «afin qu'il écrive la

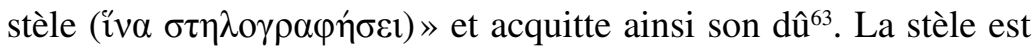
signe et signal de la puissance des dieux ${ }^{64}$. C'est pourquoi il est cohérent qu'Asclépios aussi soit une divinité jalouse de l'écriture de ses vertus ${ }^{65}$, ou que le consultant de l'oracle de Trophonios à Lébadée soit obligé d'inscrire son expérience et de l'afficher dans le sanctuaire ${ }^{66}$. Une certaine Trophimè que les dieux avaient appelée à leur service rechigna. En punition elle fut frappée de folie.

Elle a donc interrogé la Mère Tarsènè, Apollon Tarsios et Mèn d'Artemidôros Axiottènos qui règne sur Koresa, et il/elle (?) a ordonné

\section{Petzl 1994, $\mathrm{n}^{\circ} 1$}

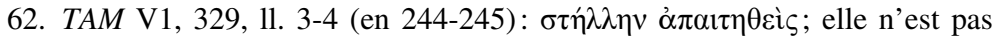
retenue par Petzl 1994 (sans doute parce que la punition n'y est pas indiquée), mais elle est retenue à juste titre par Marijana Ricl, La Conscience du péché dans les cultes anatoliens à l'époque romaine. La confession des fautes rituelles et éthiques dans les cultes méoniens et phrygiens, Belgrade, 1995, $\mathrm{n}^{\circ} 36$. Cf. aussi TAM V 1, 364 (= M. Ricl, Conscience du péché, $\mathrm{n}^{\circ} 100$ ) en 145-146 et Peter Herrmann, Hasan Malay, New Inscriptions, $\mathrm{n}^{\circ}$ 66, 11. 11-12.

63. Petzl 1994, $\mathrm{n}^{\circ} 71,1.12$.

64. Cf. Alan K. Bowman, Greg Woolf éds., Literacy and Power in the Ancient World, Cambridge, 1994. Pour les textes dits de confession, Nicole Belayche, " "Un châtiment en adviendra". Le malheur comme signe des dieux dans l'Anatolie impériale», dans Stella Georgoudi, Renée Koch Piettre \& Francis Schmidt éds., La raison des signes. Langages divinatoires, rites, signes et destin dans les sociétés de la Méditerranée ancienne, Leiden, 2011, p. 319-342.

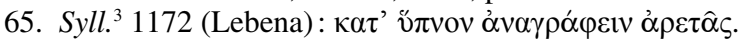

66. Pierre Bonnechère, Trophonios de Lébadée. Cultes et mythes d'une cité béotienne au miroir de la mentalité antique, Leiden-Boston (RGRW 150), 2003. 
que la puissance justicière soit inscrite sur une stèle $(\sigma \tau \eta \lambda \lambda \sigma \gamma \rho \alpha \varphi \eta \theta \hat{\eta} v \alpha \iota$ vé $\mu \varepsilon \sigma ı v)[\ldots]$ (Petzl 1994, n 57$)$.

La stèle inscrite entre dans le processus expiatoire tout en sanctionnant la fin de l'expérience ${ }^{67}$. Pollion, une fois la faute et la punition relatées, poursuit:

Il a levé [la punition] par un triphônon - une taupe, un moineau et un petit thon - et la libération de leur colère ( $\tau \eta \eta \eta v 0 v \mu \nu \lambda v \sigma i \alpha v)$, les

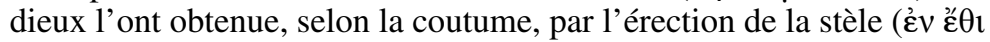

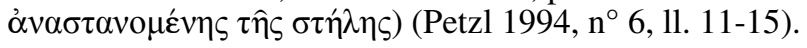

Un autre texte (Petzl 1994, $\left.n^{\circ} 8,11.3-5\right)$, tout mutilé soit-il, laisse néanmoins comprendre que les dieux ont puni une famille, car elle

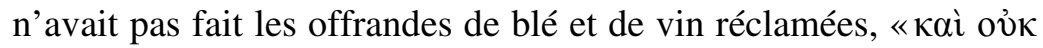

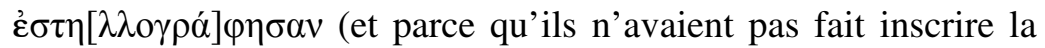
stèle)». Enfin, pour clore cette mise en série, en 263-264, Athénaios, coupable d'une faute par ignorance, fut

sommé dans un songe, après avoir reçu beaucoup de châtiments, d'élever une stèle et j'y ai inscrit les puissances du dieu. En action de grâce, j'ai fait écrire sur la stèle (Petzl 1994, nº 11) ${ }^{68}$.

Le texte célèbre les pouvoirs divins, successivement punitifs après l'infraction, puis bienveillants et miséricordieux une fois que le fidèle a manifesté sa piété. Les dieux, c'est-à-dire les prêtres, choisissent clairement le camp de la literacy pour faire connaître l'étendue de leurs pouvoirs, indépendamment du degré d'alphabétisation de leurs ouailles. Ce n'est pas l'émission qui compte, mais la réception, comme le rappelait $P$. Veyne pour un autre langage, celui des images : «ce qui importe est moins le contenu du message que la relation qu'il établit avec autrui ${ }^{69}$. En l'occurrence, l'objet-stèle inscrit assure à lui seul la glorification du dieu. Mutatis mutandis pour ces documents de facture et d'audience locale, cette fonction «pragmatique» de l'écriture est comparable à celle des grandes inscriptions impériales publiques, étudiées par J. Elsner: «what was meaningful was the monumental nature of the text itself as writing $»^{70}$. Zeus lui-même

67. Cf. CMRDM I, $\mathrm{n}^{\circ} 57$.

68. Cf. aussi Peter Herrmann, Hasan Malay, New Inscriptions, $\mathrm{n}^{\circ} 46$ (en 288289).

69. Paul Veyne, «Lisibilité des images, propagande et apparat monarchique dans l'Empire romain», Revue historique 304, 2001, p. 9.

70. Jaś Elsner, «Inventing Imperium: texts and the propaganda of monuments 
le dit dans une inscription où il est mis en scène, peut-être dans un simulacre de procès joué par les prêtres :

[...] J'ai eu Zeus pour défenseur.

[Zeus]: «Vois! Je l'ai aveuglé pour ce qu'il a commis, mais à présent, il a apaisé les dieux et en le gravant sur la stèle, il a effacé ses fautes.»

Interrogé par le conseil: «Je [le dieu] suis secourable puisque la stèle m'a été dressée au jour que j'ai prescrit» (Petzl 1994, n 5, 11. 18-24).

Les malheurs de Syntychè, qui n'avait pas rendu grâce aux dieux après l'aide qu'ils lui avaient apportée, confirment la pragmatique glorificatrice attribuée à l'écriture de la stèle :

le dieu s'irrita de cela aussi, parce que Syntychè ne le révélait pas

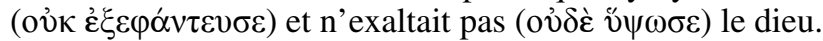

Alors, il lui intime «de dresser la nemesis (c'est-à-dire la stèle) sur son lieu (le sanctuaire ou le territoire du dieu)». Et elle signe:

Syntychè [...] qui a publié par écrit la justice divine $(\dot{\eta} \pi \rho 0 \gamma \varepsilon \gamma \rho \alpha \varphi \circ \hat{v} \sigma \alpha \tau \dot{\eta} v \text { vé } \mu \varepsilon \sigma \mathrm{v} v)^{71}$.

À l'aune d'un ordre régi par les dieux, les événements heureux et

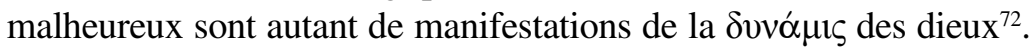
Dans le cas d'une dédicante devenue anonyme par suite de la cassure de la pierre, la punition (sous forme de quelque mal) visait à montrer (ív $\alpha \alpha^{\prime} v \alpha \delta i \xi \varepsilon l$ ), à faire connaitre publiquement les puissances des

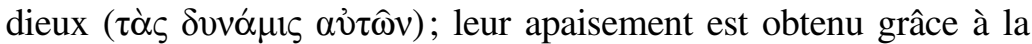

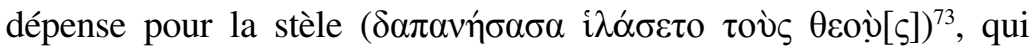
accomplit l'exigence des dieux. La divinité réclame la proclamation de sa nature infiniment supérieure et absolue, explicitement dans bien des cas, et ce sont ses pouvoirs que toutes les stèles célèbrent, par-

in Augustan Rome», dans Id. éd., Art and Text in Roman Culture, Cambridge, 1996, p. 52.

71. Petzl 1994, n 59, 11. 14-24 (cf. Georg Petzl, Hasan Malay, «A New Confession-Inscription from the Katakekaumene», Greek, Roman and Byzantine Studies 28, 1987, p. 469-471); cf. aussi Petzl 1994, nº 57 (1l. 11-12) et $\mathrm{n}^{\circ} 33$ (1l. 3-7).

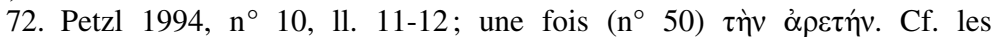
acclamations initiales, par ex. Petzl 1994, $\mathrm{n}^{\circ}$ 62: «À Mèn Axiottenos et à sa puissance », une adresse qui redouble l'invocation du dieu par celle de son numen, à fins d'intensification.

73. Petzl 1994, $n^{\circ} 33$, 1l. 5-8. La datation, inhabituelle, du texte par la prêtrise pourrait confirmer un attachement particulier de la dédicante au service du sanctuaire. 
delà la variété des faits divers. La transcription sur pierre de l'aveu de la faute est un moyen pour faire éclater cette grandeur, mais elle n'en est pas la finalité. Sa finalité consiste en un exposé écrit et durable de théologie pragmatique, d'où la présence certaine des prêtres au long du processus, même s'ils sont rarement mentionnés.

L'érection de la stèle inscrite entre dans le processus propitiatoire, mais elle dépasse cette exigence contractuelle pour servir surtout d'exaltation du dieu dans et par l'écriture. C'est pourquoi, les textes entassés dans les sanctuaires, renforcés par les reliefs d'orants, contribuent à exhorter les fidèles qui viennent auprès du dieu. Ils diffusent une «catéchèse », écrite en toutes lettres dans certains cas, par exemple au sanctuaire d'Apollon Lairbènos ${ }^{74}$. Les parénèses finales du type:

Il [le dédicant] conjure tous les hommes qu'il ne faut pas mépriser

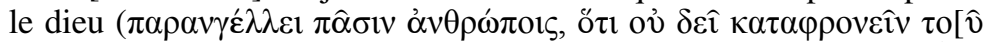

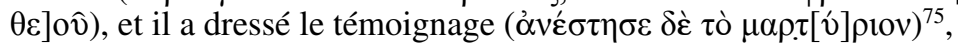

inscrivent la toute-puissance des dieux, assortie parfois de réglementations rituelles ou morales ${ }^{76}$ et représentée en miroir par l'image du dévot en orant. Cette fonction apologétique est certainement la plus originale au sein de la documentation religieuse païenne de l'Anatolie impériale. La stèle est un ع̌ $\xi \varepsilon v \pi \lambda$ ov (selon le mot latin transcrit) ${ }^{77}$, un exemplum rappelant à toutes et à tous l'ordre des dieux capables de manifestation ${ }^{78}$. Celle de Babou, fille de Manas, s'affiche comme «un modèle ( $(j o ́ \delta \varepsilon v \gamma \mu \alpha)$ pour les autres dans ce lieu »; elle appelle le bonheur sur les gens (laos) venant au sanctuaire $^{79}$. La fonction apologétique est une extension cohérente avec le but général de ces textes: glorifier, exalter les dieux. La

74. Marijana Ricl, «CIG4142-A Forgotten Confession-Inscription from NorthWest Phrygia», Epigraphica Anatolica 29, 1997, p. 42, a relevé l'importance de «the "educational motive"», mais elle le relie à la procédure pénitentielle («the confessional ritual», p. 43), alors qu'il reflète plutôt l'état transformé du fidèle.

75. Petzl 1994, $\mathrm{n}^{\circ} 9$ et les dédicaces à Apollon Lairbènos (sanctuaire connu archéologiquement), par ex. $\mathrm{n}^{\circ}$ 106. Sur le sanctuaire, Tullia Ritti, Celal Simsek, H. Yildiz, «Dediche e KАТАГРАФАI dal santuario frigio di Apollo Lairbenos », Epigraphica Anatolica 32, 2000, p. 3-6.

76. Par ex. Petzl 1994, $\mathrm{n}^{\circ} 27$.

77. Petzl 1994, $\mathrm{n}^{\circ} 120,1.8$; voir aussi n 111 (11. 8-9), 112 (1. 9) et 121 (1. 5), où la graphie est plus barbare.

78. Petzl 1994, $\mathrm{n}^{\circ} 118,11.3-4$.

79. Marijana Ricl, «CIG 4142 - A Forgotten Confession-Inscription from North-West Phrygia», p. 37, 11. 11-13 \& 18-20. 
punition dûment notifiée dans le texte est, avant même l'eulogie sur laquelle ils se terminent, la première manière de montrer à la fois la puissance des dieux et leur sollicitude.

Le but de la stèle inscrite est la louange de la grandeur du dieu, généralement acclamé au début du texte. Il n'est pas «l'aveu de sa faute... et son repentir», comme l'écrivait F. Cumont ${ }^{80}$; ou plus exactement, la fonction de l'aveu n'est pas pénitentielle, mais eulogique : il est une forme d'exposé de la grandeur des dieux. C'est pourquoi, je préfère identifier ces documents comme des stèles d'exaltation ou d'ostension ${ }^{81}$, qui à la fois constituent et figurent, en mots et en images, autant d'arétalogies des dieux, comme A.D. Nock en avait déjà eu l'intuition ${ }^{82}$.

\section{L'ÉCRITURE PERFORMATIVE DANS L'APPEL À LA JUSTICE DES DIEUX}

L'écriture ne se limite pas à exalter les dieux. Dans le cours du rituel, elle a une fonction performative bien attestée dans les pratiques juratoires et magiques. Cette fonction, déjà étudiée pour les pratiques magiques notamment, n'est pas rare dans les textes dits de confession.

Dans les différends rapportés sur les stèles, les dieux sont souvent appelés comme arbitres ou témoins dans la procédure juratoire destinée à faire éclater le bon droit ${ }^{83}$. Leur puissance est activée par un rituel que les inscriptions désignent comme l'érection du sceptre $^{84}$. L'activation de ce symbole de leur puissance et de leur

80. Franz Cumont, Les religions orientales dans le paganisme romain, Paris, $1929^{4}$, p. 36.

81. Nicole Belayche, «Résumé 2002-2003». Richard Gordon, «Raising a sceptre», p. 196, conclut de même: «texts whose raison d'être is to glorify the god».

82. Arthur D. Nock, Essays on Religion and the Ancient World, I, Oxford, 1972, p. 427, n. 77, rangeait ces inscriptions dans «the aretalogy type in general»; Vincenzo Longo, Aretalogie nel mondo greco. I. Epigrafi e papiri, Genova, 1969, en a d'ailleurs retenu cinq dans son corpus, $\mathrm{n}^{\circ}$ 80-84.

83. Ou appelés en recours après l'échec d'une conciliation à l'amiable, Petzl $1994, n^{\circ}$ 68. Sur la question de la justice divine, Angelos Chaniotis, «'Tempeljustiz' im kaiserzeitlichen Kleinasien», p. 353-384 et Id., «Under the Watchful Eyes of the Gods: Divine Justice in Hellenistic and Roman Asia Minor», dans Stephen Colvin éd., Greco-Roman East. Politics, Culture, Society, Yale Classical Studies 31, 2004, p. 1-43.

84. Richard Gordon, «Raising a sceptre», Nicole Belayche, «Du texte àl'image : les reliefs sur les stèles "de confession” d'Anatolie», dans Sylvia Estienne, 
pouvoir justicier se retrouve également dans des imprécations funéraires gravées ${ }^{85}$, destinées à protéger durablement l'intégrité

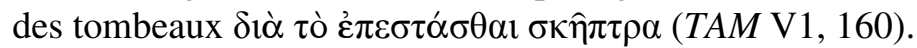

Si quelqu'un a détruit ou détériore cela (= ce tombeau), qu'il encoure la colère des dieux; pour cela que soit faite l'imprécation par

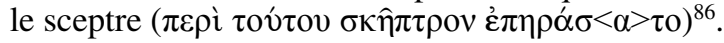

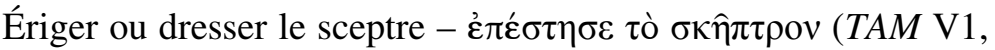
231) - est un geste rituel performatif qui met le dieu en présence, le sceptre étant investi de puissance dans le champ du serment depuis Homère (Il. I, 223-246 \& X, 319-332). Une inscription de 166-167 relie explicitement activation du sceptre, serment et écriture dans le processus rituel:

Alors que Sardion avait juré et s'était parjurée, pour cette raison puisqu'elle était mineure - Eudoxos ayant payé 9 oboles [= 1,30 dr.] a délivré des serments (Ë่

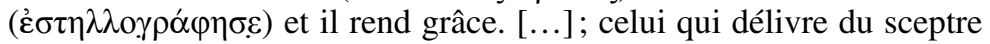
(ó $\lambda \hat{v} \omega v \sigma \kappa \hat{\eta} \pi \pi \tau \rho o v)$ donnera au sanctuaire 175 deniers et le sceptre

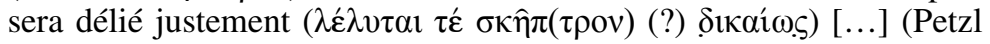
1994, n 58).

L'activation du sceptre donne aux déclarations proférées dans le temple ${ }^{87}$ valeur juratoire et elle déclenche la justice rétributive, la nemesis, des dieux tout-puissants ${ }^{88}$. Ces déclarations pouvaient être inscrites sur des tablettes ( $\pi 1 \tau \tau \alpha ́$ kiov ${ }^{89}$ qui diffèrent des pinakes qui sont des ex-voto ${ }^{90}$.

Dominique Jaillard \& Claude Pouzadoux éds., Image et religion dans l'Antiquité gréco-romaine, Naples (Collection du Centre Jean Bérard 28), 2008, p. 189-192, et Georg Petzl, «Keine Szepter an Gräbern», Zeitschrift für Papyrologie und Epigraphik 177, 2011, p. 123-126.

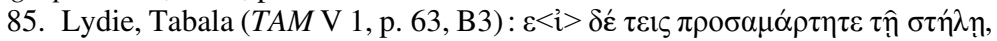

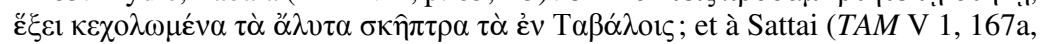

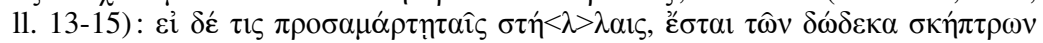

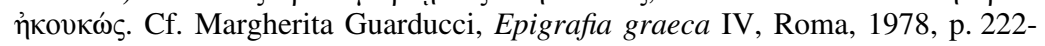
$239(«$ Arai $)$.

86. Lydie (Sattai), SEG 34, 1231; cf. Johan Strubbe, Imprecations against Desecrators, $\mathrm{n}^{\circ} 55$ et Georg Petzl, «Bemerkungen zu Inschriften aus Maionia und Saittai», Epigraphica Anatolica 6, 1985, p. 73 («er [le constructeur de la tombe] hat hierüber beim Skeptron seinen Fluch getan»).

87. Par ex. Petzl 1994, $\mathrm{n}^{\circ} 69$.

88. Cf. la réponse de l'oracle de Delphes à Glaukos fils d'Épikydès, dans Hérodote VI, 86.

89. Petzl 1994, n 60, 11. 5-7.

90. Cf. Hasan Malay \& Mustafa Hamdi Dayar, «A new confession to Zeus

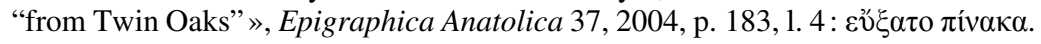


À Mèn Axiottènos. Alors que [= parce que] Hermogénès fils de Glykon et Nitonis, fille de Philoxénos, avaient volé (?) Artémidoros sur le vin, Artémidoros a donné une tablette ( $\pi \imath \tau \alpha \dot{\alpha} \kappa ı v ~ \varepsilon ̌ \delta \omega \kappa \varepsilon v)$. Le dieu a puni Hermogénès et ce dernier a apaisé le dieu et, désormais, il a de bonnes pensées $(=\text { il rend grâce })^{91}$.

Il est probable que Tatias, accusée par des villageois d'avoir empoisonné son beau-fils, avait déposé une tablette auprès du dieu :

Cette même Tatias posa le sceptre ( $\dot{\varepsilon} \pi \dot{\varepsilon} \sigma \tau \eta \sigma \varepsilon v \sigma \kappa \hat{\eta} \pi \tau \rho o v)$ et déposa

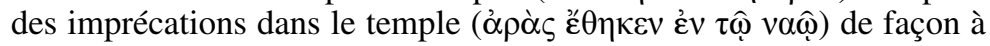
se défendre (contre une allégation) au sujet de sa dénonciation dans un tel crime ${ }^{92}$.

Selon le schéma attendu, après que Tatias a été punie dans la personne de son fils, ses petits-enfants ont délié son parjure par l'écriture de la puissance des dieux, désormais apaisés (11. 32-34):

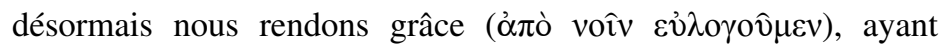
inscrit sur une stèle les puissances des dieux ( $\sigma \tau \eta \lambda \lambda \sigma \gamma \rho \alpha \varphi \eta ́ \sigma \alpha \nu \tau \varepsilon \zeta \tau \grave{\alpha} \varsigma$ $\delta v v \alpha ́ \mu ı \varsigma \tau \hat{\omega} v \theta \varepsilon \hat{\omega} v)$.

L'appel à la justice des dieux par des serments ou des incantations proférées et déposées par écrit auprès du dieu n'est pas propre aux stèles dites de confession. Il est caractéristique de la performativité des defixiones magiques, tout spécialement étudiées par Hank Versnel dans un article sur les imprécations et le droit. En Italie du Sud, une tablette de bronze fut inscrite par une certaine Kollyra: elle dédie aux servantes de la déesse les trois pièces d'or que Mellita lui a volées, si justice lui est rendue: déesse ${ }^{93}$.

et qu'elle ne respire pas librement avant de les avoir dédiées à la

91. Petzl 1994, $\mathrm{n}^{\circ} 60$.

92. Petzl 1994, $\mathrm{n}^{\circ}$ 69, 11. 9-13 (en 156-157); la traduction d'Angelos Chaniotis, 11. 11-13, est une interprétation: «as defending herself against an imputation, although she was conscious (of her guilt)».

93. Auguste Audollent, Defixionum tabellae, Paris, 1904, $\mathrm{n}^{\circ} 212=I G$ XIV 644 ; traduction Hank S. Versnel, «Les imprécations et le droit», Revue historique de droit français et étranger 65, 1987, p. $13 \mathrm{n}^{\circ} 7$ (développé dans Id., «Beyond Cursing: The Appeal to Justice in Judicial Prayers», dans Chistopher A. Faraone, Dirk Obbink éds., Magika Hiera. Ancient Greek Magic and Religion, New YorkOxford, 1991, p. 60-95). Cf. aussi Marijana Ricl, «The Appeal to Divine Justice in the Lydian Confession-Inscriptions », dans Elmar Schwertheim éd., Forschungen in Lydien, Bonn, 1995 (Asia Minor Studien 17), p. 67-76, et Angelos Chaniotis, «'Tempeljustiz' im kaiserzeitlichen Kleinasien». 
Ce parallèle démontre que le rôle des prêtres est encore plus assuré dans le recours à l'imprécation écrite, ne serait-ce que pour avoir accès au lieu sacré du dépôt, comme aujourd'hui à la portion du mur occidental du temple de Jérusalem qui subsiste.

\section{CONCLUSION : L'ACTION DE GRÂCE ARÉTALOGIQUE MISE PAR ÉCRIT}

Dans les témoignages gratulatoires, «le pinax n'est pas admirable de grandeur, mais la divinité, oui (ov̉ $\mu \varepsilon^{\prime} \gamma \varepsilon\left[\theta\right.$ o]s $\pi^{\prime}$ ivakos

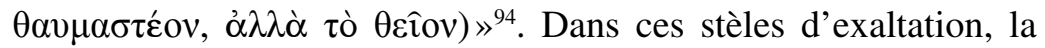
stèle est admirable de grandeur (quelle que soit sa facture, souvent soignée d'ailleurs), car elle est l'image même de la grandeur de la divinité. Les écritures étudiées n'ont rien du pouvoir mystérieux de signes sacrés (hiéro-glyphes) ou de l'aura qu'elles confèrent à leurs scribes dans d'autres expériences religieuses, égyptiennes par exemple ${ }^{95}$. Elles ressortissent d'abord du prestige qu'on accordait à la chose écrite sur support dur, à mi-chemin entre parole officielle

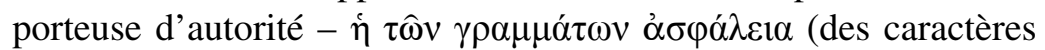
irrécusables $)^{96}$ - et témoignage de culture, de paideia ${ }^{97}$. Dans ces villages ruraux, où l'analphabétisme était sûrement majoritaire, elle a pour partie les mêmes ressorts que l'epigraphic habit dans les sociétés civiques ${ }^{98}$. Car ce sont les prêtres, c'est-à-dire les élites de ces villages où le dieu est roi / basileus, qui ont choisi de magnifier le dieu par un acte d'écriture qui grave à jamais la manifestation de leur pouvoir ${ }^{99}$. Du coup la stèle n'est plus seulement un banal hommage honorifique; elle est une épiphanie permanente du dieu, démultipliée en autant d'exemplaires qu'il y avait de stèles dressées.

nicole.belayche@ephe.sorbonne.fr

94. Emma J. Edelstein, Asclepios, 423 A I, p. 221, trad. P. Veyne.

95. Par ex. Apulée, Métamorphoses XI, 22.

96. Polybe IX, 5, 2 et Diodore de Sicile XII, 13, 2.

97. Cf. la vogue des épigrammes épigraphiques qui posent le statut de leur commanditaire. Pour un parvenu, cf. IGVR 166 (M. Antonius Gaionas).

98. WilliamV. Harris, Ancient Literacy, p. 35: «a "second-hand" literacy ».

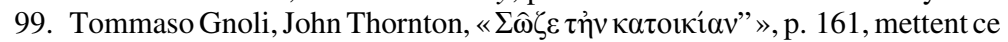
phénomène en relation avec le dynamisme de l'économie rurale. 